\title{
Black Holes and U-duality in Diverse Dimensions
}

\author{
María A. Lledó \\ Dipartimento di Fisica, Politecnico di Torino, \\ Corso Duca degli Abruzzi 24, \\ 10129 Torino, Italy. \\ INFN, Sezione di Torino.
}

ABstract: In this paper we review some properties of BPS black holes of supergravities with $n=32,16$ supersymmetries. The BPS condition, a condition on the eigenvalues of the central charge matrix, can be shown to be $U$-duality invariant. We explicitely work out $D=4, N=8$ and $D=5, N=4$ supergravities.

\section{Preliminaries}

The purpose of this work is to investigate some properties of black hole solutions of different supergravity theories in terms of the corresponding U-duality group.

Black holes are solutions of supergravity theories with point-like sources. They are rotationally invariant and are, in general, charged with respect to the vector fields of the theory. We will consider extremal black hole solutions, that is, solutions on which some of the supersymmetry charges $Q$ are null. In this case, the solutions are parametrized by the set of charges $q^{\Lambda}$ and the values of the asymptotic values of the scalar fields $\phi^{i}$ of the theory at $\infty$ (moduli).

We consider the $N$-extended super Poincaré algebra in a $D=4, \ldots 9$ dimensional space-time (we follow the notation of Ref. [1] $2 n+1$ the irreducible representation of the Clifford algebra $\mathcal{C}(1, D-1)$, of dimension $2^{n}$, is also irreducible with respect to $\operatorname{so}(1, D-1)$. This is the only spinor representation and consequently it is self-conjugated. For $D=9$ it is real, and the anticommutator of two supercharges is given by

$$
\left\{Q_{\alpha i}, Q_{\beta j}\right\}=\left(\Gamma_{\mu} C p^{\mu}\right)_{\alpha \beta} \delta_{i j}+C_{\alpha \beta} Z_{i j}
$$

where $i, j=1, \ldots N, \alpha, \beta=1, \ldots 2^{4} . C$ is the charge conjugation matrix that in this case is symmetric, so the central charge extension of the super Poincaré algebra, $Z_{i j}$, is also symmetric.
For $D=5,7$ the spinor representation is pseudoreal, so the anticommutator is instead

$$
\left\{Q_{\alpha i}, Q_{\beta j}\right\}=\left(\Gamma_{\mu} C p^{\mu}\right)_{\alpha \beta} \Omega_{i j}+C_{\alpha \beta} Z_{i j},
$$

where $\Omega$ is the symplectic bilinear form. For $D=$ $5, C=-C^{T}$, so $Z$ is antisymmetric and for $D=$ $7 C=C^{T}$ so $Z$ is symmetric.

For $D=2 n$ the irreducible representation of the Clifford algebra $\mathcal{C}(1, D-1)$ splits into two irreducible pieces under so $(1, D-1),\left(\mathbf{2}^{n-1}\right)_{ \pm}$. The projectors on each piece are

$$
\mathcal{P}_{ \pm}=\frac{1 \pm \Gamma_{D+1}}{2}, \quad Q_{ \pm}=\mathcal{P}_{ \pm} Q
$$

For $D=4,8$ these representations are complex and pairwise conjugate. The anticommutator is

$$
\begin{aligned}
\left\{Q_{\alpha i+}, Q_{j-}^{\dot{\beta}}\right\} & =\left(\mathcal{P}_{+} \Gamma_{\mu} C p^{\mu}\right)_{\alpha}^{\dot{\beta}} \delta_{i j} \\
\left\{Q_{\alpha i+}, Q_{\beta j+}\right\} & =\left(\mathcal{P}_{+} C\right)_{\alpha \beta} Z_{i j} .
\end{aligned}
$$

When $D=4, C=-C^{T}$, so $Z$ is antisymmetric and when $D=8, C=C^{T}$ and $Z$ is symmetric.

Finally, for $D=6$ the two spinor representations are pseudoreal and independent. This means that one can choose independently the number of supersymmetry charges with chirality + or $-\left(N_{+}\right.$and $\left.N_{-}\right)$.

$$
\begin{aligned}
& \left\{Q_{\alpha i+}, Q_{\beta j+}\right\}=\left(\mathcal{P}_{+} \Gamma_{\mu} C p^{\mu}\right)_{\alpha \beta} \Omega_{i j} \\
& \left\{Q_{\alpha i+}, Q_{\beta j-}\right\}=\left(\mathcal{P}_{+} C Z_{i j}\right)_{\alpha \beta} \\
& \left\{Q_{\alpha i-}, Q_{\beta j-}\right\}=\left(\mathcal{P}_{-} \Gamma_{\mu} C p^{\mu}\right)_{\alpha \beta} \Omega_{i j} .
\end{aligned}
$$


There are some rotations of the charge vectors $Q_{\alpha i}$ that leave invariant the momentum term in the anticommutator of supercharges. These transformations are automorphisms of the super Poincaré algebra, and they form the $R$-symmetry group of the algebra. It is a compact group that we will denote by $H$. The nature of this group obviously depends on the reality properties the spinors. For $D=4, \ldots 9$ these are

\section{$R$-symmetry group $H$}

$$
\begin{aligned}
& D=9 \mathrm{SO}(\mathrm{N}) \\
& D=8 \mathrm{SU}(\mathrm{N}) \times \mathrm{U}(1) \\
& D=7 \mathrm{USp}(\mathrm{N}) \\
& D=6 \mathrm{USp}\left(N_{+}\right) \times \mathrm{USp}\left(N_{-}\right) \\
& D=5 \mathrm{USp}(\mathrm{N}) \\
& D=4 \mathrm{SU}(\mathrm{N}) \times \mathrm{U}(1)
\end{aligned}
$$

We consider theories with a maximal number of supesymmetries, $n=32$. From the symmetry properties of $Z$ stated above, it follows that the central charge transforms in the following representation of the $R$-symmetry group,

\section{Central charge representation of the $R$-symmetry}

$D=9 \quad 3$ of $\mathrm{SO}(2)$, ( real symmetric tensor).

$D=8 \quad 3(+)$ of $\mathrm{SU}(2) \times \mathrm{U}(1)$, (complex triplet).

$D=7 \quad \mathbf{1 0}$ of $\mathrm{USp}(4)$, (real symmetric tensor).

$D=6 \quad 16$ of $\mathrm{USp}(4) \times \mathrm{USp}(4)$, (bispinor $(4,4)$ of $\mathrm{O}(5) \times \mathrm{O}(5))$.

$D=5 \quad \mathbf{2 7}$ of $\operatorname{USp}(8),(\Omega$-traceless symplectic antisymmetric tensor).

$D=4 \quad \mathbf{2 8}$ of $\mathrm{SU}(8)$, (complex antisymmetric tensor).

We will see that it is always possible to put the central charge matrix $Z_{i j}$ in normal form (diagonal or skew diagonal) using a transformation of the $R$-symmetry group (we denote the eignevalues by $Z_{A}$ ). Then, using the theory of induced representations, one can go to the rest frame $(p=(m, 0, \ldots, 0)$ and the supercharge anticommutator becomes

$$
\begin{aligned}
& \left\{S_{A}, S_{B}^{\dagger}\right\} \propto\left(m+Z_{A}\right) \delta_{A B}, \\
& \left\{\hat{S}_{A}, \hat{S}_{B}^{\dagger}\right\} \propto\left(m-Z_{A}\right) \delta_{A B},
\end{aligned}
$$

so we obtain a Clifford algebra. It is clear that if $m=\left|Z_{A}\right|$ for some $A$ (in general one has $\left.m \geq\left|Z_{A}\right|\right)$, there is one pair of oscillators in the Clifford algebra that decouple. Representations with such value of the central charge are representations in which certain supersymmetry charges become trivial . They are called BPS states. If all eigenvalues are equal, half of the oscillators decouple and we say that we have a representation with $\frac{1}{2} \mathrm{BPS}$. For less restrictive conditions one obtains representations with $\frac{1}{4} \mathrm{BPS}$ or $\frac{1}{8}$ BPS.

The corresponding supergravity theories can be obtained by compactifying $D=11, N=1$ supergravity on a torus $T^{d+1}$ or compactifying $D=10$, type IIA and IIB supergravity on a torus $T^{d}$. The scalar fields of the theory parametrize a coset manifold of the form $G / H$, where $G$ is a non compact group whose maximally compact subgroup is $H$. For any $d, G=E_{d+1(d+1)}[2]$ (the factor $\mathrm{U}(1)$ for $D=4$ must be supressed to have $H$ as a subgroup of $G$ ). $G$ is called the $U$-duality group. $G$ acts on the non linear manifold of the scalars, and the vector fields also transform in a representation of $G$. This representation naturally extends (1. table for each case [2ind,

$$
\begin{array}{cl}
\text { Central charge representation of the } \\
\\
\begin{array}{ll}
U \text {-duality group } \\
D=9 & \mathbf{2 + 1} \text { of } \mathrm{E}_{2}=\mathrm{SL}(2) \times \mathrm{O}(1,1) \\
D=8 & \mathbf{( 3 , 2 )} \text { of } \mathrm{E}_{3}=\mathrm{Sl}(3) \times \mathrm{Sl}(2) \\
D=7 & \mathbf{1 0} \text { of } \mathrm{E}_{4}=\mathrm{Sl}(5) \\
D=6 & \mathbf{1 6} \text { of } \mathrm{E}_{5}=\mathrm{O}(5,5) \\
D=5 & \mathbf{2 7} \text { of } \mathrm{E}_{6(6)} \\
D=6 & \mathbf{5 6} \text { of } \mathrm{E}_{7(7)}
\end{array}
\end{array}
$$

Let $\Phi^{i}$ be the scalar fields which parametrize $G / H$ and consider a local section on the principal bundle $G$ over the base $G / H, L(\phi) \in G$ (coset representative). The action of $G$ can be expressed as

$$
L\left(\phi_{g}\right)=g L(\phi) h\left(\phi_{g}\right)
$$

where $h\left(\phi_{g}\right) \in H$ is a transformation in the fiber over $\phi_{g}$. Choosing a representation of $G$ the coset 
representative becomes a matrix $L_{a}^{\Lambda}(\Phi)$. The indices $a, \Lambda$ run over the same represenation of $G$ (and of $H$ ), the different names used to remind the transformation rule (11.7).

The charges of a black hole solution can be computed by integrating the Hodge dual of the field strengths of the vector fields present in the theory on a $D-2$ spacial surface enclosing the source. We denote these charges by $q^{\Lambda}$. Then, the central charges of the black hole solutions are given by

$$
Z_{a}(q, \Phi)=q_{\Lambda}^{T} L_{a}^{\Lambda}(\Phi)
$$

Notice that the vectors fields are in a certain representation of $G$, which necesarily coincides with

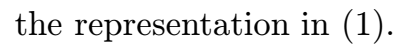

When the central charges correspond to BPS states, some supersymmetry generators are null when acting on the black hole solutions, so the solutions are "supersymmetric". The condition to be a BPS black hole is a condition on $Z$, that is, on $q$ and $\Phi$. Due to the form of $(1.81)$, it is clear that any condition $E_{\alpha}(Z)=0$ (where $\alpha$ runs over some representation $T$ of $G$ ) that is covariant under $G$, that is

$$
E_{\alpha}(Z g)=E_{\beta}(Z) T(g)_{\alpha}^{\beta},
$$

will become simply a condition on $q, E_{\alpha}(q)=0$. It was shown in [3in that this condition is actually moduli independent or $U$-duality invariant in all theories in dimensions $D=4, \ldots, 9$ with $n=32,16$ supersymmetries. Here we will review these results, in particular $D=4, N=8$ and $D=5, N=4$.

\section{Maximal supergravity in dimen- sions $D=4, \ldots, 9$}

We study the diagonalization of the matrix of central charges in the different cases (Table $\left.\underline{1}_{1}^{1}\right)$. A matrix is quaternionic (or symplectic) if

$$
Z^{*}=-\Omega Z \Omega \text {. }
$$

A matrix satisfying this conditions can be understood as a matrix whose entries are quaternions, and these are in the representation

$$
\mathrm{Id}_{2 \times 2}, \quad i \sigma_{1}, \quad i \sigma_{2}, \quad i \sigma_{3} .
$$

One has the following results:

1. Any matrix can be brought to a diagonal form by making a transformation

$$
Z_{D}=U_{1} Z U_{2}^{\dagger}
$$

where $U_{i}$ are orthogonal matrices if $Z$ is real, unitary if $Z$ is complex and unitary symplectic if $Z$ is quaternionic (In the representation of $n \times n$ quaternionic matrices by complex, $2 n \times 2 n$ matrices, it is $\Omega Z$ which is brought to diagonal form). 2 . In the case that the original matrices are symmetric, hermitian or symplectic hermitian respectively, $U_{1}=U_{2}$ and the eigenvalues are real. 3. An antisymmetric matrix can be brought to skew diagonal form by a transformation

$$
Z_{S D}=U Z U^{T}
$$

where $U$ belongs to the appropriate group as before and the eigenvalues are real.

For $D=9$ we can trivially diagonalize $Z$ with an $R$-symmetry transformation by using the result 2. For $D=8$, an $R$-transformation $U$ brings the matrix $Z$ to $U Z U^{T}$. It is easy to see that to diagonalize the $2 \times 2$ matrix it is enough to use the result 1 with $U_{2}^{\dagger}=U_{1}^{T}$, being the eigenvalues real. For $D=7, Z$ is four dimensional and since the spinors are pseudoreal it is quaternionic. Since it is also symmetric, we can take advantage of the isomorphism $\mathrm{Sp}(4) \approx \mathrm{O}(5)$ and decompose $Z$ as

$$
Z_{i j}=Z_{I J}\left(\gamma^{I J}\right)_{a b}, I J=1, \ldots 5
$$

where $Z^{I J}$ is real and antisymmetric and

$$
\gamma^{I J}=\frac{1}{2}\left[\gamma^{I}, \gamma^{J}\right]
$$

and $\gamma^{I}$ are the gamma matrices of $\mathrm{O}(5)$. We can skew diagonalize $Z^{I J}$ using the result 3 , with only two independent eigenvalues. For $D=6$ one can use the result 1 and diagonalize the matrix with an element $\left(U_{1}, U_{2}\right) \in \mathrm{USp}(4) \times \operatorname{USp}(4)$. The quaternionic property is preserved by a transformation $Z_{D}=U_{1} Z U_{2}^{\dagger}$, but since the eigenvalues are not real there are four independent real quantities. Finally, for $D=5,4$ one can use the result 3.

For $D=7,8,9$ there are only two independent eigenvalues. In the generic situation, when 


$\begin{array}{lllll}D & N & H & \mathrm{Z} & \text { eigenvalues } \\ 9 & 2 & \mathrm{SO}(2) & \text { real symmetric. } & 2 \\ 8 & 2 & \mathrm{SU}(2) \times \mathrm{U}(1) & \text { complex symmetric } & 2 \\ 7 & 4 & \mathrm{USp}(4) \approx \mathrm{O}(5) & \text { quaternionic symmetric } & 2 \\ 6 & 4,4 & \mathrm{USp}(4) \times \mathrm{USp}(4) & \text { quaternionic } & 4 \\ 5 & 8 & \mathrm{USp}(8) & \text { quaternionic antisymmetric } & 4 \\ 4 & 8 & \mathrm{SU}(8) & \text { complex antisymmetric } & 4\end{array}$

Table 1: Central charge matrix

the two eigenvalues are different, we have that the bound $m=\left|Z_{A}\right|$ can be reached only by the highest eigenvalue. Then, $\frac{1}{4}$ of the oscillators of the supersymmetry alegra are null on the solution. We say that we have a $\frac{1}{4}$ BPS state. If all the eigenvalues are equal, the half of the oscillators decouple and we have a $\frac{1}{2}$ BPS state. For $D=4,5,6$ we have four eignevalues and the generic case, when all of them are different preserves $\frac{1}{8}$ of the supersymmetry. If the eigenvalues are equal by pairs we have $\frac{1}{4}$ BPS states and if they are all equal we have $\frac{1}{2}$ BPS states. Exotic cases like having only one pair of equal eigenvalues and the rest different or having three equal eigenvalues and a different fourth one are forbidden on physical grounds as we will see in the next example.

\subsection{BPS states in $D=4, N=8$ Supergrav-} ity.

As we have seen in this case $Z$ is a $8 \times 8$ complex antisymmetric matrix, so it can be skew diagonalized

$$
Z_{S D}=U Z U^{T}
$$

with 4 independent eigenvalues. Since we can use only transformations $U \in \mathrm{SU}(8)$, the eigenvalues are not real. Instead there is an overall phase that cannot be removed [i $\left[\begin{array}{l}4 \\ 1\end{array}\right]$. In fact, this phase is an extra parameter of the solution which usually is set to zero and all the eigenvalues real. Their absolute value, can be computed as the square root of the eigenvalues of the matrix $Z Z^{\dagger}$.

$\frac{1}{2}$ BPS Since all the eigenvalues are real, the matrix $Z Z^{\dagger}$ is a multiple of the identity,

$$
Z Z^{\dagger}=\operatorname{Tr}\left(Z Z^{\dagger}\right) \frac{1}{8} \mathrm{Id}
$$

This is an $\mathrm{SU}(8)$ covariant constraint. The idea is to find an $\mathrm{E}_{7,7}$ covariant constraint that neces- sarily implies (2.1). Then we will be in the situation (1.9i), where the $\frac{1}{2}$ BPS condition is moduli independent. Nevertheless, the eigenvalues will depend on the moduli.

We consider the irreducible representation of $\mathrm{E}_{7,7} 56$ (the number indicates the dimensionality of the representation). Under $\mathrm{SU}(8)$ it decomposes as $\mathbf{5 6}=\mathbf{2 8}+\overline{\mathbf{2 8}} . \quad \mathbf{2 8}$ is the twofold antisymmetric representation of $\mathrm{SU}(8)$ in which $Z$ sits. We will write a vector of $\mathbf{5 6}$ symbolically as $\tilde{Z}=(Z, \bar{Z})$. We consider now the quartic invariant of this representation of $\mathrm{E}_{7,7}$ [י1,

$$
I=4 \operatorname{Tr}(Z \bar{Z})^{2}-(\operatorname{Tr} Z \bar{Z})^{2}+2^{4}(\operatorname{Pf} Z+\operatorname{Pf} \bar{Z}),
$$

where

$$
\operatorname{Pf} Z=\frac{1}{2^{4} 4 !} \epsilon^{A B C D R P G H} Z_{A B} Z_{C D} Z_{R P} Z_{G H}
$$

is the Pfaffian of $Z$. Let us take the second derivative of this invariant

$$
\frac{\partial^{2} I}{\partial \tilde{Z} \partial \tilde{Z}} .
$$

It is a quadratic polynomial which is a symmetric tensor, so it sits in the $(\mathbf{5 6} \times \mathbf{5 6})_{S}=\mathbf{1 5 9 6}$ representation of $\mathrm{E}_{7}$ which is reducible and decomposes as $1463+133.133$ is the adjoint of $\mathrm{E}_{7}$, so we can take the projection

$$
\left.\frac{\partial^{2} I}{\partial \tilde{Z} \partial \tilde{Z}}\right|_{A d j_{E_{7}}} .
$$

Since 133 decomposes as $\mathbf{6 3 + 7 0}$ under SU(8) (63 is the adjoint representation of $\mathrm{SU}(8)$ ), the expression (2.3i) splits into two $\mathrm{SU}(8)$ covariant polynomials

$$
\begin{aligned}
& V_{A}^{C}=\left.\frac{\partial^{2} I}{\partial Z_{A B} \bar{\partial} Z^{C B}}\right|_{A d j_{S U(8)}} \approx \\
& \left(Z_{A B} \bar{Z}^{C B}-\frac{1}{8} \delta_{A}^{C} Z_{P Q} \bar{Z}^{P Q}\right),
\end{aligned}
$$




$$
\begin{aligned}
& V_{[A B C D]}^{+}=\left.\frac{\partial^{2} I}{\partial Z_{A B} \bar{\partial} Z^{C B}}\right|_{\mathbf{7 0}} \approx \frac{\partial^{2} I}{\partial Z_{[A B} \partial Z_{C D]}}- \\
& \frac{1}{4 !} \epsilon^{A B C D P Q R S} \frac{\partial^{2} I}{\left.\partial \bar{Z}^{[A B} \partial \bar{Z}^{C D]}\right]} .
\end{aligned}
$$

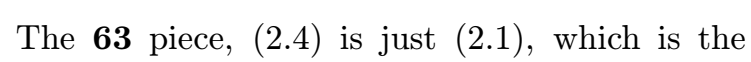
constraint we want. But the $\mathbf{7 0}$ part $V_{[A B C D]}^{+}$is not, in principle, zero. Here is where the $G / H$ structure of the theory enters. Consider $L(\Phi)$ : $G / H \mapsto G$, the local section or coset representative of $G / H$. Using this map we can make a pull-back of the Maurer-Cartan left invariant forms on $G$ to an open set of $G / H$,

$$
\Omega(\phi)=L^{-1}(\phi) d L(\phi)=\omega_{i} T_{i}+P_{\alpha} T_{\alpha} .
$$

$T_{i} \in \mathcal{H}$ are the generators in the Lie algebra of $H$ and $T_{\alpha} \in \mathcal{K}$, where $\mathcal{G}=\mathcal{H}+\mathcal{K}$ is a Cartan decomposition of $\mathcal{G}$, the Lie algebra of $G$. $\omega_{i}$ are the components of the spin connection of the bundle $G \mapsto G / H$ and $P_{\alpha}$ is the vielbein of the invariant metric on $G / H$.

In the case of $\mathrm{E}_{7,7} / \mathrm{SU}(8)$, the equation (2.6i) takes the form

$$
\nabla_{S U(8)} Z_{A B}=\frac{1}{2} \epsilon_{A B C D} \bar{Z}^{C D} .
$$

Taking the covariant derivative $\nabla_{S U(8)}$ of the equation $V_{A}^{C}=0$ and using the Maurer-Cartan equations above, one obtains

$$
V_{+}^{A B C D}=0
$$

as we wanted to show.

Notice that the reality of the eigenvalues follows in fact from (2. 2.5$)$, so it follows from the $\mathrm{E}_{7,7}$ invariance.

$\frac{1}{4}$ BPS. In this case we have that the eignevalues are equal by pairs. It can be shown [3in that the $\mathrm{E}_{7,7}$ covariant condition for this to happen is

$$
\frac{\partial I}{\partial Z_{A B}}=0 \quad\left(\Rightarrow \frac{\partial I}{\partial \bar{Z}^{A B}}=0\right),
$$

where $I$ is again the quartic invariant. The reality also follows from the $\mathrm{E}_{7,7}$ invariance.

$\frac{1}{8} \mathrm{BPS}$ is the generic case when all the eigenvalues are different. One can always make the highest eigenvalue (which is the mass of the BPS state) real, so no equation is needed to assure reality. Having three or two eigenvalues equal and the rest different makes the quartic invariant negative 阿. Since this invariant has an interpretation as the entropy of the black hole squared, $I \propto S^{2}$ it cannot be negative, so these cases are excluded.

For all the other theories in Table ${ }_{1}^{1}$ a similar

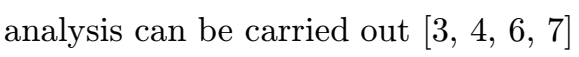

\section{Supergravities with 16 supersym- metries. BPS states of $D=5$, $N=4$ supergravity}

Theories with sixteen supersymmetries are obtained as compactifications of the heterotic string on tori $\mathrm{T}^{d}(1 \leq d \leq 6)$. They can also be obtained by compactifying $D=11$ supergravity or $D=10$ Type IIA and IIB supergravity on manifolds preserving less supersymmetries, as $\mathrm{K}_{3}$. In these theories there are matter fields and the $U$-duality group $G$ depends on the matter content as well as on the dimension of spacetime. The maximal compact subgroup is a direct product $H_{R} \times H_{M}$ where $H_{R}$ is the R-symmetry of the corresponding supersymmetry algebra and $H_{M}$ is the group acting on the matter multiplets. If $n$ is the number of matter multiplets this group is $H_{M}=\mathrm{O}(n)$. $G$ is of the form $\mathrm{O}(10-$ $D, n) \times \mathrm{O}(1,1)$ for $5 \leq D \leq 9$ while for $D=4$ it is $\mathrm{SL}(2) \times \mathrm{O}(6, n)$. The R-symmetry groups are $\mathrm{O}(10-D)$ for $5 \leq D \leq 9$ and $\mathrm{O}(6) \times \mathrm{O}(2) \approx \mathrm{SU}(4) \times \mathrm{U}(1)$ for $D=4$.

The $G$ and $H_{R}$ representations of the central charges are given in the following tables.

\section{Central charge representation of $H_{R}$.}

$$
\begin{array}{rrrl}
D=9 & \mathbf{1} & \mathrm{O}(1)=\mathrm{Id} \\
D=8 & \mathbf{1}^{\mathbf{c}} \text { complex } \mathrm{U}(1) \approx \mathrm{O}(2) \\
D=7 & \mathbf{3} \text { real } & \mathrm{SU}(2) \approx \mathrm{USp}(2) \\
D=6 & \mathbf{4} \text { real } & \mathrm{O}(4) \approx \mathrm{USp}(2) \times \mathrm{USp}(2) \\
D=5 & \mathbf{1}+\mathbf{5} \text { real } & \mathrm{O}(5) \approx \mathrm{USp}(4) \\
D=4 & \mathbf{6}^{\mathbf{c}} \text { complex } \mathrm{O}(6) \times \mathrm{O}(2) \approx \\
& & \mathrm{SU}(4) \times \mathrm{U}(1)
\end{array}
$$

From the above table, and according to our previous analysis, it follows that the matrix of central charges, $Z$, has only one independent real eigenvalue for $D=6, \ldots 9$ and two independent 
eigenvalues for $d=5,6$. Therefore, for $D=$ $6, \ldots 9$ only $1 / 2$ BPS states can occur while for $D=4,5$ both, $1 / 2$ and $1 / 4$ BPS states can occur.

\section{Central charge representation of $G$}

$$
\begin{array}{rl}
D=6, \ldots 9 & \mathbf{d}+\mathbf{n} \text { real vector } \\
& \mathrm{O}(d, n) \times \mathrm{O}(1,1) \\
D=5 & \mathbf{1}+(\mathbf{5}+\mathbf{n})(\text { singlet+vector }) r \\
& \mathrm{O}(5, n) \times \mathrm{O}(1,1) \\
D=4 & (\mathbf{2}, \mathbf{6}+\mathbf{n}) \\
& \mathrm{Sl}(2) \times \mathrm{SO}(6, n)
\end{array}
$$

We will briefly outline here the case of $D=$ 5. It corresponds to heterotic string on $T^{5}$ or $D=11$ supergravity on $K^{3} \times T^{2}$. The number of matter multiplets is $n=21$, although our analysis is independent of such number.

The central charge $\hat{Z}$ is an antisymmetric quaternionic matrix. This implies that the ma$\operatorname{trix} Z=\hat{Z} \Omega$ is hermitian and quatenionic. The $4 \times 4$ matrix depends on 6 real parameters. We want to exploit the isomorphism $\mathrm{O}(5) \approx \mathrm{USp}(4)$, so we decompose $Z$ as

$$
Z=Z^{a} \gamma_{a}+Z^{0} \mathrm{Id},
$$

where $\gamma_{a}, a=1, \ldots 5$ are the $\mathrm{O}(5) \gamma$-matrices and $Z^{a}, Z^{0}$ are real numbers. $Z^{0}$ is a singlet under $\mathrm{O}(5)$ and $Z^{a}$ is a vector. It follows that

$$
\begin{aligned}
& \operatorname{Tr} Z=4 Z^{0} \\
& (\operatorname{det} Z)^{1 / 2}=\frac{1}{8}(\operatorname{Tr} Z)^{2}-\frac{1}{4} \operatorname{Tr} Z^{2}= \\
& Z^{0^{2}}-\vec{Z}^{2}
\end{aligned}
$$

The characteristic equation of $Z$ (or better, its square root) is

$$
\begin{aligned}
& \sqrt{\operatorname{det} Z-\lambda \mathrm{Id}}= \\
& \lambda^{2}-\frac{1}{2} \operatorname{Tr} Z \lambda+(\operatorname{det} Z)^{1 / 2}=0,
\end{aligned}
$$

implying that $Z$ has two coinciding eigenvalues (in absolute value) either if

$$
\operatorname{Tr} Z=0 \quad \text { or } \quad \frac{1}{4}(\operatorname{Tr} Z)^{2}=4(\operatorname{det} Z)^{1 / 2}
$$

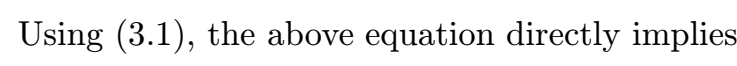

$$
Z_{0} Z_{a}=0
$$

The eigenvalues are given by

$$
\lambda_{1,2}=\frac{1}{2}\left(\frac{1}{2} \operatorname{Tr} Z \pm \sqrt{\operatorname{Tr} Z^{2}-\frac{1}{4}(\operatorname{Tr} Z)^{2}}\right),
$$

being the plus sign the mass squared of the BPS state.

The central charge representation of $G$ (and $H)$, singlet + vector, is reducible and the coset representative $L_{a}^{\Lambda}$ splits into blocks

$$
\left(\begin{array}{cc}
e^{2 \sigma} & \\
& e^{-\sigma}(M)
\end{array}\right)
$$

where $\sigma$ parametrizes $\mathrm{O}(1,1)$ and $M$ is in the fundamental representation of $\mathrm{O}(5, \mathrm{n})$. Since $Z_{a}=$ $q_{\Lambda} L_{a}^{\Lambda}$, then $Z_{0}=e^{2 \sigma} q_{0}$. The condition $Z_{0}=0$ implies $q_{0}=0$, which is a singlet of $\mathrm{O}(5+\mathrm{n})$, and then, $U$-duality invariant.

If $Z_{I}, I=1, \ldots n$ are the matter charges associated to the $n$ matter multiplets, we have that, because of $(2 \cdot 6 i)$,

$$
\nabla_{O(5)} Z_{a}=\operatorname{Tr}\left(\gamma_{a} P_{I}\right) Z^{I}+Z_{a} d \sigma,
$$

therefore $Z_{a}=0$ implies $Z_{I}=0$. This is also an $\mathrm{O}(5, \mathrm{n})$ invariant statement since, it comes by differentiating the quadratic invariant polynomial

$$
I=\sum_{a=1}^{5} Z_{a} Z^{a}-\sum_{I=1}^{M} Z_{I} Z^{I} .
$$

Therefore, $Z_{a}=Z_{I}=0$ implies $q^{\Lambda}=0$ where $q^{\Lambda}, \Lambda=1, \ldots 5+n$, is a fixed charge vector of $\mathrm{O}(5, \mathrm{M})$, as found in [īi].

\section{Acknowledgments}

I want to thank to my collaborators R. D'Auria and S. Ferrara for reading the manuscript an suggesting many improvements.

\section{References}

[1] J. Strathdee, Int. Jour. Mod. Phys. A 2 (1987) 273.

[2] E. Cremmer, in Supergravity '81, eds. S. Ferrara and J. G. Taylor, p. 313;

B. Julia in Superspace and Supergravity, eds. S. W. Hawking and M. Rocek (Cambridge, 1981), p. 331.

[3] R. D'Auria, S. Ferrara and M. A. Lledó, Phys. Rev. D 60 (1999) 084007-1. 
[4] S. Ferrara and J. Maldacena, (19) Class. Quant. Grav. 15 (1998) 749.

[5] S. Ferrara, Int. Jour. Mod. Phys. A 133791.

[6] S. Ferrara and M. Gunaydin Int. Jour. Mod. Phys. A13 (1998) 2075.

[7] H. Lu, K. S. Stelle and C. N. Pope, Class. Quant. Grav. 15 (1998) 537.

[8] L. Andrianopoli, R. D'Auria and S. Ferrara, Phys. Lett. B403 (1997) 12.

[9] R. Kallosh and B. Kol, Phys. Rev. D53 (1996) 5344. 